\title{
Plasma pNfH differentiate SBMA from ALS
}

Lombardi Vittoria PhD ${ }^{* 1,2}$, Bombaci Alessandro MD ${ }^{* 2,3}$, Zampedri Luca ${ }^{1}$, Lu Ching-Hua MD, $\mathrm{PhD}^{1,4}$, Malik Bilal PhD ${ }^{5}$, Zetterberg Henrik $\mathrm{PhD}^{1,7,8,9}$, Heslegrave Amanda $\mathrm{PhD}^{1,7}$, Rinaldi Carlo MD, $\mathrm{PhD}^{6}$, Greensmith Linda $\mathrm{PhD}^{1}$, Hanna Michael FRCP ${ }^{1}$, Malaspina Andrea MD, $\mathrm{PhD}, \mathrm{FRCP} \mathrm{P}^{2}$ and Fratta Pietro MD, $\mathrm{PhD}^{1}$

${ }^{1}$ Institute of Neurology, University College London Institute of Neurology, Queen Square, London, WC1N 3BG, UK. ' Blizard Institute, Queen Mary, University of London, UK. " "Rita Levi Montalcini" Department of Neuroscience, University of Torino, Turin, Italy. ${ }^{4}$ Department of Neurology, China Medical University Hospital, Taiwan. ${ }^{5}$ Basildon Hospital, Basildon, UK. ${ }^{6}$ Department of Paediatrics, University of Oxford, Oxford, OX1 3QX, UK. ${ }^{7} U K$ Dementia Research Institute at UCL, London WC1E 6BT, UK. ${ }^{8}$ Clinical Neurochemistry Laboratory, Sahlgrenska University Hospital, S-431 80 Mölndal, Sweden. ${ }^{9}$ Department of Psychiatry and Neurochemistry, Institute of Neuroscience and Physiology, the Sahlgrenska Academy at the University of Gothenburg, S-431 80 Mölndal, Sweden.

* These authors contributed equally to this work

\section{Address for correspondence:}

Dr Pietro Fratta

University College London Institute of Neurology,

Queen Square, London, WC1N 3BG, UK.

Tel: +44 (0)2034483899 Fax: +44 (0)2043383111

Email:p.fratta@ucl.ac.uk

and

Dr Andrea Malaspina

Queen Mary University

4 Newark St, London, E1 2AT, UK.

Email: a.malaspina@qmul.ac.uk

Title Character count: 6

Text word count: 991

Figures: 1

References: 11

Financial Disclosures:

All authors report no disclosures

Study Funded by the NIHR UCLH Biomedical Research Centre Grant \#BRC279566 


\section{Introduction}

Spinal and bulbar muscular atrophy (SBMA), known as Kennedy disease (KD), is a slowly progressive adult-onset X-linked neuromuscular disorder with no effective treatment. It is characterized by progressive limb and bulbar muscle weakness, associated with metabolic and endocrine alterations ${ }^{1,2}$. SBMA is caused by the expansion of a CAG repeat in exon 1 of the androgen receptor $(A R)$ gene; more than 37 repeats are pathogenic ${ }^{1}$. Whilst the genetic test is diagnostic, biomarkers would aid the initial differential diagnosis, and furthermore, there is a strong need for disease activity and progression markers to inform effective clinical trials design.

Neurofilaments ( $\mathrm{Nfs}$ ), both light and heavy chains, are now becoming a widely accepted marker of neuronal damage and a prognostic biomarker for amyotrophic lateral sclerosis (ALS) and other neurodegenerative disease ${ }^{3,4,5,6,7}$. Recently, plasma neurofilament-light-chain $(\mathrm{NfL})$ levels were unexpectedly found not to be raised in SBMA patients ${ }^{8}$. This finding supports other lines of evidence, including an increase in plasma muscle damage markers, myopathic changes in biopsies and a series of genetic experiments in mouse models, that point to a primary myopathic involvement in $\mathrm{SBMA}^{2,9,10}$.

We here used the highly sensitive Simoa platform to investigate plasma levels of phosphorylated neurofilament-heavy-chain $(\mathrm{pNfH})$, another well-established marker of neuronal damage, in SBMA patients and in a rodent model of disease.

\section{Materials and Methods}

We have undertaken cross-sectional pNfH analysis using the Single Molecule Array (SIMOA) platform in plasma from 46 SBMA patients, 50 ALS patients (25 ALS-Fast and 25 ALS-Slow, as previously described ${ }^{8}$ ) and 50 healthy controls (HCs) previously tested for $\mathrm{NfL}$. Participant demographic and clinical data are summarized in Figure 1A, and detailed methods and statistical analysis are listed in Supplementary material.

Sera from SBMA (AR100) and wild type littermate controls ( $\mathrm{N}=10$ for each group; 18 months) were also investigated. Ethical approval was obtained from the East London and the City Research Ethics Committee (09/H0703/27) and the Ethical Review Panel of UCL Institute of Neurology (PPL PE83401B1). 


\section{Results}

Plasma pNfH levels were unchanged in SBMA compared to HCs (Figure 1B). These results were also confirmed in SBMA mice, where serum pNfH levels were even lower in AR 100 compared to WT mice ( $p=0.009)$ (Figure 1C). Conversely, in both fast- and slow-progressing ALS groups there was a statistically significant increase of plasma pNfH levels compared to HCs (ALS-Slow $p<0.001$; ALS-Fast $p<0.0001$ ), conforming with previous reports ${ }^{3,5}$. Plasma pNfH levels differed between ALS-Fast and ALS-Slow patients (Mann-Whitney test $p=0.012$, significance was not retained after Dunn's multiple comparison correction). A weak but significant correlation between $\mathrm{pNfH}$ plasma levels and disease progression rate to last visit $(P R L){ }^{11}$ was observed $\left(r_{s}=0.36, p=0.01\right)$, supporting $p N f H$ as a possible marker of disease progression rate in ALS. No correlation was found between $\mathrm{pNfH}$ and ALSFRSr (ALS Functional-Rating-Scale revised), SBMAFRS (SBMA Functional-Rating-Scale) and AMAT (Adult-Myositis-Assessment-Tool) scales in SMBA patients $\left(r_{s}=-0.03, p=0.86\right.$; $r_{s}=0.03, p=0.89 ; r_{s}=0.02, p=0.89$, respectively) or between $p N f H$ and ALSFRS $r$ in ALS patients $\left(r_{s}=-0.19, p=0.18\right)$. pNfH plasma levels did not correlate with age.

Importantly, pNfH plasma levels were significantly different between SBMA and both ALS subgroups $(p<0.0001)$. A ROC curve was highly significant ( $A \cup C=0.95, p<0.0001$; Figure 1E) and identified the cut-off point of plasma pNfH that most effectively distinguishes SBMA from ALS as 105 pg/ml (highest Youden-Index, 98\% sensitivity, 86\% specificity).

Lastly, we compared our $\mathrm{pNfH}$ results to measurements of $\mathrm{NfL}$ previously obtained on the same samples ${ }^{8}$. Cox regression analysis, showed a significant correlation between pNfH and NfL levels ( $r_{s}=0.77, p<0.0001$; Figure 1D).

\section{Discussion}

Contrary to what would be expected in a motor cell disorder ${ }^{3,8,11,7}$, this study does not show a disease-related increase of $\mathrm{pNfH}$ in SBMA patients. This finding was also confirmed in a well-established SBMA mouse model. Conversely, and in addition to what previously reported, $\mathrm{pNfH}$ levels increase in ALS and correlate with disease progression rate ${ }^{11}$.

We were able to identify a robust cut-off level $(A \cup C=0.95)$ of $\mathrm{pNfH}$ that could distinguish SBMA from ALS. Although the diagnosis of SBMA has a firm genetic ground and 
it is compounded by robust clinical and neurophysiological observations, measurement of $\mathrm{Nfs}$, which rise in the prodromal phase and in early stages of $\mathrm{ALS}^{12}$, may help orientate the diagnostic approach at weakness onset, particularly when only signs of lower motor neuron involvement are present. In this context, absence of a rise in Nf levels would make consideration for genetic testing mandatory. Our data also suggest that, differently form ALS, Nfs may be of limited use in SBMA clinical trials.

Limits of this study are represented by the cross-sectional design that does not allow us to infer about the variation of $\mathrm{pNfH}$ during the disease course. With regard to the analytical aspect, in this study we employed one of the most sensitive platforms for neurofilament analysis; this approach was the same used for the NfL study ${ }^{8}$. Although previous work has highlighted the inherent difficulties encountered in $\mathrm{pNfH}$ measuring in biological fluids ${ }^{11}$, we show here a good correlation of $\mathrm{pNfH}$ and $\mathrm{NfL}$ expression levels in the same samples, possibly as a result of the sensitive immunodetection method employed.

Besides, the finding that $\mathrm{pNfH}$ is not increased in SBMA supports the recent discovery of normal level of $\mathrm{NfL}$ in SBMA, although it was not obvious. $\mathrm{pNfH}$ and $\mathrm{NfL}$ are two isoforms of the $\mathrm{Nf}$ core: the first involved in the cell structure homeostasis and axonal transport, the second the most abundant and essential component of the core. Anyway, we believe that there is no basis to prefer $\mathrm{pNfH}$ over $\mathrm{NfL}$ as a biomarker of axonal damage due to concerns of sample stability.

Lack of increase of $\mathrm{Nf}$, both $\mathrm{NfL}$ and $\mathrm{pNfH}$, in SBMA, traditionally considered a lower motor neuron disease, is surprising. Although the difference with ALS could be accounted for by the different progression and aggressiveness of the disorder, the finding of increased $\mathrm{Nfs}$ in peripheral neuropathies ${ }^{3,5}$ suggests progression rate cannot fully explain this discrepancy. Recent work on patients' muscle biopsies have identified primary myopathic changes in SBMA, and different experiments on disease models have also suggested that the primary muscle changes could be the main driver of the neuromuscular phenotype ${ }^{2,8,10}$. Our results support this view, although they do not exclude a role for neuronal loss. 
In conclusion, plasma pNfH concentrations are not increased in SBMA patients and in a mouse model of disease, as opposed to ALS. These results suggest $\mathrm{pNfH}$ could be a useful biomarker in the differential diagnosis between SBMA and ALS.

\section{Acknowledgements}

We thank the patients involved in the study and their families for the participation and support to KD research. All samples were obtained from the ALS Biomarker study. PF is supported by an MRC/MNDA LEW Clinician Scientist Fellowship and the NIHR UCLH BRC. This study was supported by the NIHR UCLH BRC, the UCL Kennedy's Disease Fund and KDUK. CR is funded by a Welcome Trust Clinical Research Career Development Fellowship and the Muscular Dystrophy Association (MDA). HZ is funded by the UK Dementia Research Institute at UCL, the European Research Council, the Swedish Research Council and the Knut and Alice Wallenberg Foundation. The Simoa instrument was bought using a Welcome Trust multi-user equipment grant (PI: $\mathrm{HZ})$.

\section{References:}

1. Querin G, Sorarù G, Pradat PF. Kennedy disease (X-linked recessive bulbospinal neuronopathy): A comprehensive review from pathophysiology to therapy. Rev Neurol (Paris). 2017;173(5):326-337. doi:10.1016/j.neurol.2017.03.019

2. La Spada A. Spinal and Bulbar Muscular Atrophy. University of Washington, Seattle; 1993. http://www.ncbi.nlm.nih.gov/pubmed/20301508. Accessed February 7, 2019.

3. Steinacker P, Feneberg E, Weishaupt J, et al. Neurofilaments in the diagnosis of motoneuron diseases: A prospective study on 455 patients. J Neurol Neurosurg Psychiatry. 2016;87(1):12-20. doi:10.1136/jnnp-2015-311387

4. Rosengren LE, Karlsson J-E, Karlsson J-O, Persson LI, Wikkels $\varnothing$ C. Patients with Amyotrophic Lateral Sclerosis and Other Neurodegenerative Diseases Have Increased Levels of Neurofilament Protein in CSF. J Neurochem. 2002;67(5):2013-2018. doi:10.1046/j.14714159.1996.67052013.x

5. Feneberg E, Oeckl P, Steinacker $P$, et al. Multicenter evaluation of neurofilaments in early symptom onset amyotrophic lateral sclerosis. Neurology. 2018;90(1):e22-e30. doi:10.1212/WNL.0000000000004761

6. C.-H. L, C. M-W, E. G, et al. Neurofilament light chain: A prognostic biomarker in amyotrophic lateral sclerosis. Neurology. 2015;84(22):2247-2257. doi:10.1212/WNL.0000000000001642 
7. Darras BT, Crawford TO, Finkel RS, et al. Neurofilament as a potential biomarker for spinal muscular atrophy. Ann Clin Transl Neurol. 2019;6(5):932-944. doi:10.1002/acn3.779

8. Lombardi V, Querin G, Ziff OJ, et al. Lombardi V et al. Neuron and muscle biomarkers for SBMA. :1-12.

9. Manzano R, Sorarú G, Grunseich C, et al. Beyond motor neurons: expanding the clinical spectrum in Kennedy's disease. J Neurol Neurosurg Psychiatry. 2018;89(8):808-812. doi:10.1136/jnnp-2017-316961

10. Dahlqvist JR, Oestergaard ST, Poulsen NS, Thomsen C, Vissing J. Refining the spinobulbar muscular atrophy phenotype by quantitative MRI and clinical assessments. Neurology. 2019;92(6):e548-e559. doi:10.1212/WNL.0000000000006887

11. Lu C-H, Petzold A, Topping J, et al. Plasma neurofilament heavy chain levels and disease progression in amyotrophic lateral sclerosis: insights from a longitudinal study. I Neurol Neurosurg Psychiatry. 2015;86(5):565-573. doi:10.1136/jnnp-2014-307672

12. Benatar M, Wuu J, Andersen PM, Lombardi V, Malaspina A. Neurofilament light: A candidate biomarker of presymptomatic amyotrophic lateral sclerosis and phenoconversion. Ann Neurol. 2018;84(1):130-139. doi:10.1002/ana.25276 
A

\begin{tabular}{|l|c|c|c|c|c|c|c|c|c|}
\hline $\begin{array}{c}\text { Patients } \\
\text { Group }\end{array}$ & $\mathbf{n}^{\circ}$ & $\begin{array}{c}\text { Sex } \\
\mathbf{M} / \mathrm{F}\end{array}$ & $\begin{array}{c}\text { Age } \pm \\
\text { SD } \\
(\mathbf{y})\end{array}$ & $\begin{array}{c}\mathbf{n}^{\circ} \mathrm{CAG} \\
\pm \mathrm{SD}\end{array}$ & $\begin{array}{c}\text { PRL } \pm \\
\text { SEM }\end{array}$ & $\begin{array}{c}\mathrm{pNfH} \pm \\
\text { SEM } \\
(\mathbf{p g} / \mathrm{ml})\end{array}$ & $\begin{array}{c}\text { ALSFRSr } \pm \\
\text { SEM }\end{array}$ & $\begin{array}{c}\text { SBMAFRS } \pm \\
\text { SEM }\end{array}$ & $\begin{array}{c}\text { AMAT } \pm \\
\text { SEM }\end{array}$ \\
\hline SBMA & 46 & $46 / 0$ & $\begin{array}{c}56,6 \pm \\
11,1\end{array}$ & $\begin{array}{c}42.1 \pm \\
0.4\end{array}$ & N/A & $42 \pm 4.9$ & $\begin{array}{c}38.8 \pm 0.8 \\
(\mathrm{n}=37)\end{array}$ & $\begin{array}{c}41.1 \pm 1.3 \\
(\mathrm{n}=36)\end{array}$ & $\begin{array}{c}31.8 \pm \\
1.4 \\
(\mathrm{n}=38)\end{array}$ \\
\hline ALS-Slow & 25 & $14 / 11$ & $\begin{array}{c}66,9 \pm \\
12,7\end{array}$ & $\mathrm{~N} / \mathrm{A}$ & $\begin{array}{c}0.27 \pm \\
0.04\end{array}$ & $\begin{array}{c}629 \pm \\
159\end{array}$ & $37.6 \pm 1.6$ & $\mathrm{~N} / \mathrm{A}$ & $\mathrm{N} / \mathrm{A}$ \\
\hline ALS-Fast & 25 & $10 / 15$ & $\begin{array}{c}66.4 \pm \\
10.7\end{array}$ & $\mathrm{~N} / \mathrm{A}$ & $\begin{array}{c}1.61 \pm \\
0.12\end{array}$ & $\begin{array}{c}1615 \pm \\
327\end{array}$ & $31.7 \pm 1.8$ & $\mathrm{~N} / \mathrm{A}$ & $\mathrm{N} / \mathrm{A}$ \\
\hline HC & 50 & $17 / 33$ & $\begin{array}{c}58,5 \\
(6,8)\end{array}$ & N/A & N/A & $76 \pm 12.7$ & N/A & N/A & N/A \\
\hline
\end{tabular}

B

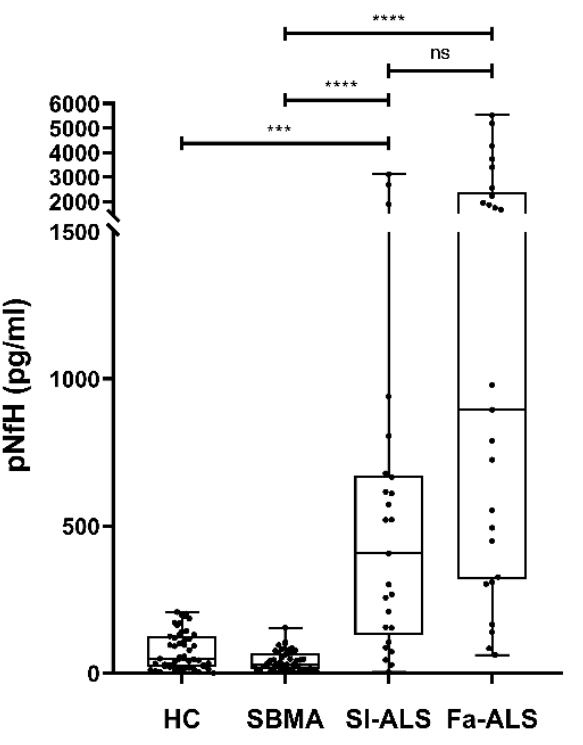

D

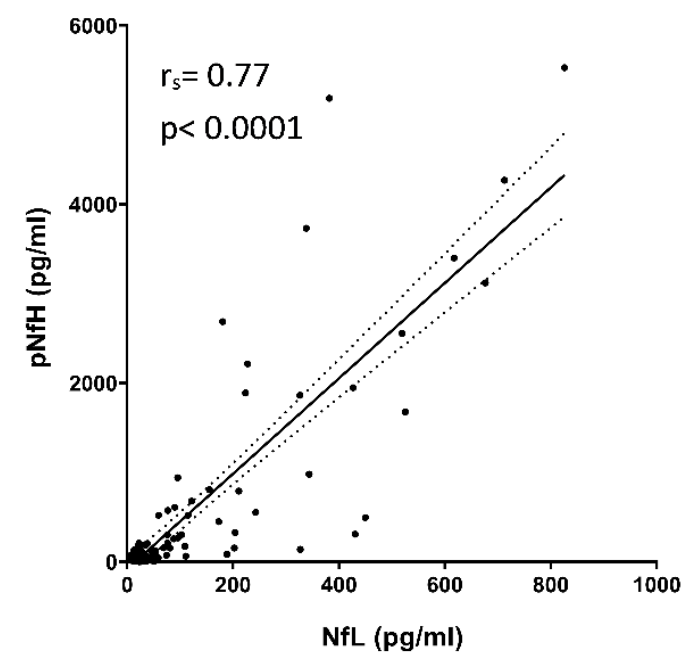

C

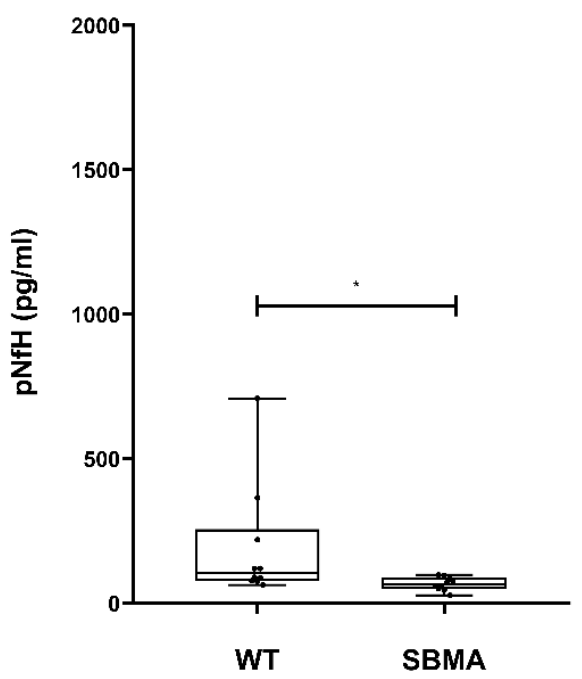

E

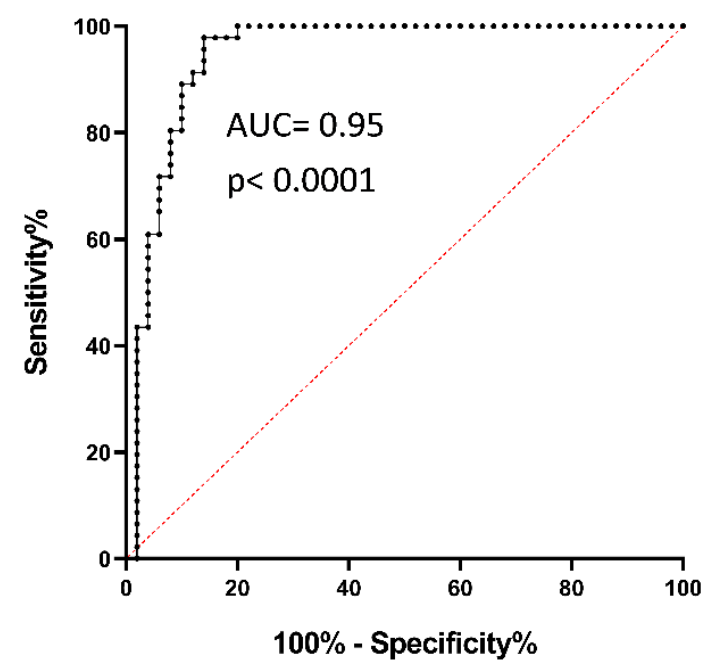




\section{Figure 1}

(A) Cohort demographic and genetic information, $\mathrm{pNfH}$ and scales values. (B) $\mathrm{pNfH}$ concentration in HCS, SBMA, ALS-Slow and ALS-Fast. Using Kruskal-Wallis test and Dunn's multiple comparison test we observed reduced levels of both $\mathrm{pNfH}$ compared to ALS groups (all $p$-value $<0.0001$ ). (C) $\mathrm{pNfH}$ levels from mice AR100 (SBMA) and littermate controls (WT) show a light statistically significant difference (Mann Whitney test). (D) Correlation between pNfH and NfL $\left(r_{s}=0.77, p<\right.$ 0.0001). (E) ROC curve, created comparing $\mathrm{pNfH}$ and $\mathrm{NfL}$ levels, shows a high AUC $(0.95 ; 95 \% \mathrm{Cl}$ $0.90-1.00$ ). The value associated to the highest Youden-Index is $105 \mathrm{pg} / \mathrm{ml}$ (98\% sensitivity, $86 \%$ specificity, PPV 0.88, NPV 0.98).

Fa-ALS= Fast-progressing amyotrophic lateral sclerosis; SI-ALS= slow-progressing amyotrophic lateral sclerosis; $\mathrm{WT}=$ wild type mice; $\mathrm{HCs}=$ Healthy controls; $\mathrm{SBMA}=$ spinal bulbar muscular atrophy; $\mathrm{pNfH}=$ phosphorylated-Heavy-Neurofilaments; $\mathrm{NfL}=$ Light-Chain-Neurofilaments; $\mathrm{Y}=$ years; $\mathrm{F}=$ Female; $\mathrm{M}=$ Male; $\mathrm{n}^{\circ}=$ Number; $\mathrm{n}^{\circ} \mathrm{CAG}=$ number of $\mathrm{CAG}$ repeats in androgen receptor gene; $\mathrm{SD}=$ standard deviation; $\mathrm{SEM}=$ standard error of the mean; $\mathrm{AUC}=$ area under curve; $\mathrm{AMAT}=$ adult myopathy assessment tool; SBMAFRS= spinal bulbar muscular atrophy function rating scale; ALSFRS $=$ amyotrophic lateral sclerosis function rating revised scale; $P R L=$ progression rate to last visit; $P P V=$ positive predictive value: $N P V=$ negative predictive value; $p=p$-value.

ns= not statistically significant result; ${ }^{*} p<0.05 ;{ }^{*} p<0.01 ;{ }^{* * *} p<0.001 ; * * * * p<0.0001$. 


\section{Materials and Methods}

\section{Study design}

We have undertaken cross-sectional $\mathrm{NfH}$ analysis using the SIMOA platform in plasma from SBMA and ALS patients, visited in Motor neuron clinics at University College of London Hospital and at Queen Mary Hospital between Sep 2009 and Nov 2017. We included 46 SBMA patients, 50 ALS patients (25 ALS-Fast and 25 ALS-Slow, as previously described ${ }^{1}$ ) and 50 healthy controls ( $\mathrm{HCS}$ ) previously tested for $\mathrm{NfL}^{1}$. basis of their disease progression rate to last visit (PRL): PRL ALS-Slow<0.6 and ALS-Fast $>0.9$. PRL was calculated as 48 (corresponding to the in-healthy state, before symptoms onset) minus the ALS FunctionalRating-Scale revised score (ALSFRSr) at the last visit, divided by time interval in months between symptoms onset and last visit date.

Sera from SBMA (AR100) and wild type littermate controls ( $N=10$ for each group; 18 months) were also investigated. Ethical approval was obtained from the East London and the City Research Ethics Committee (09/H0703/27) and the Ethical Review Panel of UCL Institute of Neurology (PPL PE83401B1).

Samples were processed, stored and analysed as previously described ${ }^{5}$. All blood samples were collected into EDTA-containing tubes, centrifuged at $20^{\circ} \mathrm{C}$ at $3.500 \mathrm{rpm}$ for 10 minutes within 1 hour and stored at $-80^{\circ} \mathrm{C}$.

We measured pNfH levels in plasma performing a single molecule array (Simoa)-based assay (Quanterix, Lexington, MA)², using pNF-Heavy Discovery Kit 102669.

\section{Clinical assessment}

SBMA patients had a genetical confirm of diagnosis, while ALS patients had a diagnosis of definite or probable ALS according to the revised El-Escorial criteria ${ }^{3}$. Disease severity was assessed using ALSFRSr ${ }^{4}$, SBMA Functional-Rating-Scale (SBMAFRS) ${ }^{5}$ and Adult-MyositisAssessment-Tool (AMAT) ${ }^{6}$ scale in SBMA patients and using ALSFRS-r scale in ALS patients. Demographic and clinical data of patients are gathered in Table 1. 


\section{Statistical analysis}

Mann-Whitney $\mathrm{U}$ test and Kruskal-Wallis tests were performed to analyse plasma $\mathrm{pNfH}$ levels between groups. Dunn's multiple comparisons test was performed following Kruskal-Wallis test in case of significant differences. Receiver operating characteristic (ROC) curve, and the corresponding sensitivity, specificity, positive and negative predictive values, accompanied by their $95 \% \mathrm{Cls}$, was performed in order to identify the best cut-off level of pNfH to separate ALS and SBMA patients.

Correlation between parameters was calculated by Spearman rank correlation $r$. The level of significance for all statistical tests was set at 0.05. The program Prism V.8 (GraphPad Software, La Jolla, CA) was used to perform statistical calculations.

\section{References:}

1. Lombardi V, Querin G, Ziff OJ et al. Neuron and muscle biomarkers for SBMA. Neurology 2019;92:1-7. doi:10.1212/WNL.0000000000007097

2. Rissin DM, Kan CW, Campbell TG, et al. Single-molecule enzyme-linked immunosorbent assay detects serum proteins at subfemtomolar concentrations. Nat Biotechnol. 2010;28(6):595-599. doi:10.1038/nbt.1641

3. Brooks BR, Miller RG, Swash M, Munsat TL, World Federation of Neurology Research Group on Motor Neuron Diseases. El Escorial revisited: revised criteria for the diagnosis of amyotrophic lateral sclerosis. Amyotroph Lateral Scler Other Motor Neuron Disord. 2000;1(5):293-299. http://www.ncbi.nlm.nih.gov/pubmed/11464847. Accessed February 7, 2019.

4. Cedarbaum JM, Stambler N, Malta E, et al. The ALSFRS-R: a revised ALS functional rating scale that incorporates assessments of respiratory function. BDNF ALS Study Group (Phase III). I Neurol Sci. 1999;169(1-2):13-21. http://www.ncbi.nlm.nih.gov/pubmed/10540002. Accessed February 7, 2019.

5. Hashizume A, Katsuno M, Suzuki K, et al. A functional scale for spinal and bulbar muscular atrophy: Cross-sectional and longitudinal study. Neuromuscul Disord. 2015;25(7):554-562. doi:10.1016/j.nmd.2015.03.008

6. Harris-Love MO, Fernandez-Rhodes L, Joe G, et al. Assessing function and endurance in adults with spinal and bulbar muscular atrophy: validity of the adult myopathy assessment tool. Rehabil Res Pract. 2014;2014:873872. doi:10.1155/2014/873872 